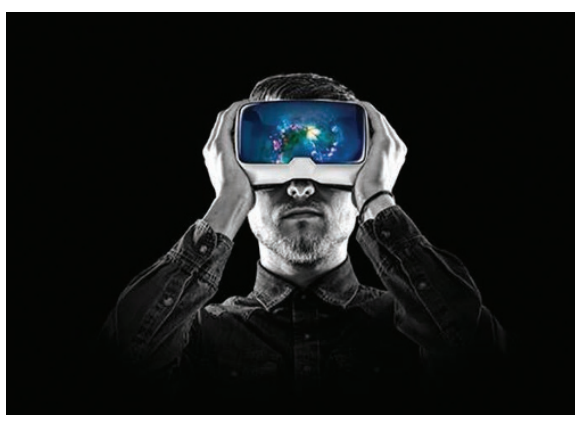

The quality of your dentistry is dependent on the products you use. For over 50 years, COLTENE has been developing world-class materials that help dental professionals improve their clinical outcomes.

Exhibiting at the BDIA Dental Showcase on stand L20, the COLTENE team will be giving delegates more information about the wide range of products they have to offer. This includes the full range of internationally renowned restorative materials, such as BRILLIANT EverGlow and Fill-Up!, as well as the latest endodontic products that COLTENE has developed in cooperation with dentists. These advanced products include the HyFlex EDM NiTi files, which - as the fifth generation of COLTENE's

\title{
Bone level tapered implant solutions
}

The Straumann Bone Level Tapered Implant features the Straumann Bone Control Design and the CrossFit connection together with its corresponding prosthetic CrossFit components from the Bone Level product portfolio.

The implant has an apically tapered and self-cutting design which, when combined with Roxolid material and SLActive surface, make it particularly suitable for anatomically and clinically challenging situations like those involving soft bone or fresh extraction sockets where higher initial stability is required.

The Straumann Bone Level Tapered Implant now includes a surgical protocol using single use drills, profile drills and taps designed for flexibility and efficiency. Single patient drills always come sterile, sharp, ready to use. After the surgery,

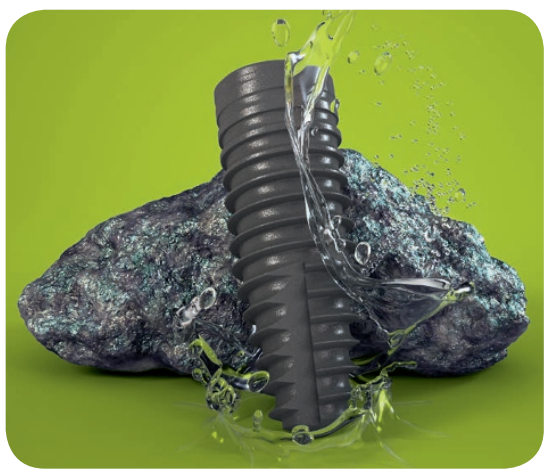

simply dispose of the single patient instruments and save your clinic the time for reprocessing and space for storage.

For more information on our range of Straumann Bone Level Tapered Implant solutions, including the new single use instruments, contact Straumann on 01293 651230 or visit www.straumann.co.uk.

\section{A little different to other dealers}

Dental Sky are a little different to other dealers. Firstly, they like to keep things simple and don't believe in confusing promotions, or reams of small print. They will never knowingly be undersold and believe in price clarity and simple offers so you know exactly what you're getting and at what price.

Secondly, they offer a broad range of sundries and are delighted to have recently been selected as the sole distributor for The Wand, a computer-assisted anaesthesia system, capable of providing virtually pain-free dentistry.

root canal files - exhibits vital properties for endodontic success.

COLTENE will also be showcasing the new Biosonic UC150 Ultrasonic Cleaning system, which features a 5.7 litre tank capacity and a low noise level of $63 \mathrm{~dB}$.

The team are eager to talk to aspiring dental professionals who want to learn more about the vast range of dental products they have to offer - and to find our more about what the profession needs from providers.

To find out more visit stand L20, go to www.coltene.com,email info.uk@coltene. com or call 01444235486 .

\section{Driving change - with your help}

The British Society of Dental Hygiene and Therapy (BSDHT) works tirelessly behind the scenes to make dental hygienists and therapists' voices heard.

Working in collaboration with the British Association of Dental Therapists (BADT), it is currently pursuing exemptions to the Medicines Act that would specify in legislation a list of prescriptiononly medicines for dental hygienists and dental therapists to administer or supply.

In the quest to enable dental hygienists and dental therapists to deliver the essential care their patients need, a successful outcome would have a huge impact on patients and the profession as a whole. But they need you to continue the momentum! To build their case, they need your stories on how the lack of prescribing responsibilities among dental hygienists and dental therapists has affected you, your patients and your practice. They also need your support to help fund the campaign as they move into the next phase.

To find out more about the work BSDHT and BADT are doing, led by respective Immediate Past-Presidents Michaela ONeill and Fiona Sandom, visit the BSDHT at the BDIA Dental Showcase this October.

For more information about the BSDHT and its campaign with BADT, visit www.bsdht.uk, call 01788575050 or email enquiries@bsdht.org.uk. To share your experiences email prescribing@ bsdht.org.uk. 